\title{
Deltamethrin-impregnated Dog Collars Have a Potent Anti- feeding and Insecticidal Effect on Lutzomyia longipalpis and Lutzomyia migonei
}

\author{
John R David/ ${ }^{+}$, Luisa M Stamm, Haroldo Sergio Bezerra*, \\ Raimundo Nonato Souza*, Robert Killick-Kendrick**, \\ José Wellington Oliveira Lima*
}

Department of Immunology and Infectious Diseases, Harvard School of Public Health, 665 Huntington Avenue, Boston, MA 02115, USA *Fundacão Nacional de Saúde, Fortaleza, CE, Brasil **Department of Biology, Imperial College at Silwood Park, Ascot, UK

Deltamethrin-impregnated PVC dog collars were tested to assess if they were effective in protecting dogs from sand fly bites of Lutzomyia longipalpis and Lu. migonei. A protective effect against Old World species Phlebotomus perniciosus was demonstrated before. Four dogs wearing deltamethrin collars and three dogs wearing untreated collars (not impregnated with deltamethrin) were kept in separate kennels for over eight months in a village on the outskirts of Fortaleza in Ceará, Brazil. Periodically, a dog from each group was sedated, placed in a net cage for $2 \mathrm{~h}$ in which 150 female sand flies had been released 10-15 min before. Lu. longipalpis were used 4, 8, 12, 16, 22, 27, and 35 weeks after the attachment of the collars. Lu. migonei were used 3, 7, 11, 15, 22, 26, and 36 weeks after attachment. During 35 weeks, only $4.1 \%$ (81 of 2,022) Lu. longipalpis recovered from the nets with the deltamethrin collared dogs were engorged, an anti-feeding effect of $96 \%$. Mortality initially was over $90 \%$ and at 35 weeks was 35\% with half of the sand flies dying in the first $2 \mathrm{~h}$. In contrast, 83\% of the 2,094 Lu. longipalpis recovered from the nets containing the untreated collared dogs were engorged and the mortality ranged from zero to $18.8 \%$ on one occasion with $1.1 \%$ dying in the first $2 \mathrm{~h}$. Similar findings were found with Lu. migonei: of 2,034 sand flies recovered over this period, only 70 were engorged, an anti-feeding effect of $96.5 \%$, and mortality ranged from $91 \%$ initially to $46 \%$ at 36 weeks. In contrast, engorgement of controls ranged from 91 to $71 \%$ and a mortality ranged from 3.5 to $29.8 \%$.

These studies show that deltamethrin impregnated collars can protect dogs against Brazilian sand flies for up to eight months. Thus, they should be useful in a program to control human and canine visceral leishmaniasis.

Key words: sand flies - deltamethrin - anti-feeding effect - Leishmania - control - visceral leishmaniasis dog collars

Visceral leishmaniasis (VL) is one of the most important vector-borne diseases in the world with 400,000 new cases and 50,000 deaths occurring each year worldwide (Murray \& Lopez 1996). In 1990, more than 2 million Disability Adjusted Life Years (DALYs) were estimated for VL in the world, second only to malaria for parasitic infections (Murray \& Lopez 1996).

\footnotetext{
This work was supported in part by USPHS NIH grant and from the Fundação Nacional de Saúde, Fortaleza, Ceará, Brazil. LM Stamm was supported by a Fulbright US student program grant.

${ }^{+}$Corresponding author. Fax: +508-748.0672. E-mail: jdavid@hsph.harvard.edu

Received 15 August 2000

Accepted 6 April 2001
}

VL is a serious health problem in the northeastern states of Brazil and in the State of Minas Gerais (Grimaldi et al. 1989, Marzochi \& Marzochi 1994). There is considerable evidence that the domestic dog is an important reservoir host (Nicolle \& Comte 1908, Adler \& Theodore1932, Bettini et al. 1986, Bettini \& Gradoni 1986, Shaw \& Lainson 1987, Ashford et al. 1998). The sand fly Lutzomyia longipalpis is the vector of the parasite, Leishmania chagasi, which is essentially indistinguishable from Le. infantum in the Old World (Mauricio et al. 2000a,b). Control of VL has been attempted by the destruction of serologically positive dogs and by spraying insecticides in peridomestic areas.

The cost of these control activities from 19841996 in Brazil exceeded \$96 million. In 1995 alone, the staff involved in the control program totaled 1,839 (Akhavan 1996). In seven years, 
nearly 1 million houses were sprayed and blood samples were taken from 6.5 million dogs for serology; 153,819 seropositive dogs were destroyed (Akhavan 1996). Despite this effort, morbidity and mortality in the human population remains unacceptably high, and the possibility of reducing the risk of infection to a negligible level is remote by the methods currently in use.

In 1997, Killick-Kendrick et al. reported that plastic dog collars impregnated with the insecticide deltamethrin protected dogs from $96 \%$ of Phlebotomus pernicious bites for up to eight months. This sand fly transmits Le. infantum, the parasite causing canine leishmaniasis (CanL) and VL in France and some other countries of the Mediterranean Basin. If the deltamethrin-impregnated collars can be shown to be effective in preventing sand flies from biting dogs in Brazil, we would have a simple procedure to control this animal reservoir host. In contrast to killing seropositive dogs, collaring dogs would be simpler and more acceptable to owners of the dogs at risk.

In this paper, we show that deltamethrin impregnated dog collars (referred to as deltamethrin collars) are effective in preventing the neotropical sand flies $\mathrm{Lu}$. longipalpis and Lu. migonei from biting dogs for at least six to eight months and, furthermore, the majority of the sand flies are killed.

\section{MATERIALS AND METHODS}

Dog collars - The collars were a gift from Hoechst Roussel Vet, Paris. They consist of a 48 $\mathrm{cm}$ strip of white polyvinylchloride (PVC) weighing $20 \mathrm{~g}$ impregnated with deltamethrin $40 \mathrm{mg} / \mathrm{g}$ and are called Scalibor ${ }^{\mathrm{TM}}$ ProtectorBands ${ }^{\mathrm{TM}}$. They caused no visible reactions on the skin of the dogs during the period of the experiments. PVC collars without deltamethrin were used as untreated controls. If the collars were large on the dogs, the loose ends were cut off.

Sand flies - Lu. longipalpis and Lu. migonei sand flies were reared in laboratory colonies at the Laboratory of Entomology at the Fundação Nacional de Saúde, Fortaleza, Ceará (CE), following a technique already described (KillickKendrick et al. 1977). The Lu. longipalpis colony originated from captures in the municipalities of Baturité and Sobral, CE, in July 1996, whereas the Lu. migonei colony originated from captures in the municipality of Maranguape, CE, in September, 1997. Briefly, larvae of both species were reared in plaster-lined pots of volume $200 \mathrm{ml}$ and were fed on rabbit feces. Three days post-emergence, females of Lutzomyia of both species were fed on anaesthetised hamsters (Tiopental, $0.025 \mathrm{~g} / \mathrm{ham}$ ster), and were maintained in mesh cages $\left(40 \mathrm{~cm}^{3}\right)$ in a B.O.D. incubator (Fanem $\left.{ }^{\circledR}\right)$. Temperature in- side the incubator was $25^{\circ} \mathrm{C}$ and humidity was 80 $90 \%$. The sand flies were stored for a period of four days for complete digestion of blood, then were placed in rearing pots, consisting of bottomless plastic pots with the wall and bottom covered by a layer of plaster of Paris. After oviposition, the females died and were removed. The pots containing the hatched larvae were placed in plastic boxes and maintained in the B.O.D incubator at the same humidity $(80-90 \%)$ and temperature $\left(25^{\circ} \mathrm{C}\right)$ as the adults. Adults were maintained in the Barraud cages $\left(40 \mathrm{~cm}^{3}\right)$ with saturated sucrose solution until the experiments. Sand flies 3-4 days old were used in all experiments, since in preliminary observations, we found that females fed well at that age. On the day of the experiment, female sand flies were counted and transferred using a Castro aspirator (Sherlock \& Pessoa 1964, Alexander 2000) into two separate larger plaster-lined pots measuring approximately $1500 \mathrm{~cm}^{3}$ and kept in styrofoam containers with moist cotton wool to keep the humidity high for transport. The females were transferred into large cubic net cages 10-15 min before the anesthetised dogs were placed inside the nets. The females found dead inside the transport pots were counted and subtracted from the total number of sand flies released into the net cages.

Cubic nets - The net cages used in the experiment to expose the dogs to the sand flies were as described (Killick-Kendrick et al. 1997) with minor modifications. They measured $1.2 \mathrm{~m}^{2}$, and were $1.8 \mathrm{~m}$ in height. The bottom and the lower $55 \mathrm{~cm}$ of the walls were made of cotton to prevent drafts while the rest of the net was made of fine netting. On one side, the net had a vertical double-headed zipper to adjust the size of the opening when releasing flies, adding the dogs or entering to capture the flies. The nets were suspended on PVC piping tied by cord to beams in the ceiling. A 6.5 $\mathrm{cm}$ thick sponge overlaid with plastic was laid under the net. The nets were placed in two indoor locations and separated by a canvas curtain. In preliminary tests these two locations were shown to give similar sand fly biting rates on dogs prior to the experiments with the collars. The temperature during the experiments varied between 24$28^{\circ} \mathrm{C}$, and humidity was $89-98 \%$. In a series of 17 preliminary experiments using $10 \mathrm{dogs}$, we found no significant difference in the biting of sand flies in this temperature and humidity range. Atmospheric pressure was also measured and was approximately $1009 \mathrm{mb}$.

Dogs - The dogs were 7-9 month-old mongrels weighing between 6-9 $\mathrm{kg}$ from the kennel at the Núcleo de Medicina Trópical, Universidade Federal do Ceará, and from the city pound in Fortaleza. All dogs were vaccinated against ra- 
bies (Tec-Par), canine viruses (Duramune, Fort Dodge, Campinas, São Paulo), and de-wormed at least four times with ivermectin and mebendazole before the start of the experiment. The kennels were located in Gereraru, approximately $25 \mathrm{~km}$ from Fortaleza. All dogs were fed the same mixture of dog food and given continuous clean water. Ten dogs were initially tested with Lu. longipalpis and Lu. migonei to assess their variability in attractiveness to sand flies. Three dogs were eliminated before experiments with the collar started because of illness. The four deltamethrin-collared dogs and three untreated-collared dogs were housed in separate kennels made of chicken wire and a roof, and were approximately $37.5 \mathrm{~m}$ apart. To avoid contamination, the kennels were cleaned with a neutral soap twice a day by different people, and handlers of the deltamethrin-collared dogs always wore disposable gloves. Dogs were walked to the experimental site, approximately $600 \mathrm{~m}$ from the kennels, by separate handlers and entered the indoor test site by different doors.

On the days of the experiments dogs were not fed before sedation. To anesthetise the dogs, atropine $(0.044 \mathrm{mg} / \mathrm{kg}$ dog's body weight, Aritson) was given intramuscularly $10 \mathrm{~min}$ before a mixture of Rompun $^{\mathrm{R}}(0.2 \mathrm{mg} / \mathrm{kg}$ dog's body weight, Bayer $)$ and $\operatorname{Ketalar}^{\mathrm{R}}(10 \mathrm{mg} / \mathrm{kg}$ dog's body weight, ParkeDavis). A second dose, $70-80 \%$ of the first dose of Rompun and Ketalar, was administered after the dogs spent $1 \mathrm{~h}$ in the net to keep them sedated for a total of $2 \mathrm{~h}$. Dogs wore disposable diapers to absorb urine during the 2-h exposure period in the net. Upon awaking, the dogs were carried back to the kennel in wheelbarrows, were allowed to recover, and were fed. The dogs in each group in the experiments were picked using a rotating schedule.

Exposure of dogs to sand flies - Pots containing 150 females of Lu. longipalpis or Lu. migonei, were used in the experiments. Male Lu. longipalpis exhibit a lek-like behavior and arrive at the source of the blood meal before the females (Jarvis \& Rutledge 1992). In some preliminary observations, we therefore added 50 males to the females in the net cages assuming this might improve female engorgement. We found, however, that the females showed an average engorgement of $64 \%$ when males were present $(n=20)$ and $81 \%$ without males $(\mathrm{n}=5), \mathrm{p}<0.025$. Therefore all experiments with collared dogs were carried out only with female flies, which were released into the cubic nets at 6 p.m. (In preliminary studies we found there was no significant difference whether the experiments started at 5:30 p.m. or 7 p.m, sunset being at 5:30 p.m, data not shown). The dogs were placed in the cages 10-15 min after the sand flies and kept there for $2 \mathrm{~h}$ in total darkness and then removed. Details of the procedures are given below. The protocol for these studies was approved by the Standing Committee on Animals of the Harvard Medical Area.

Experimental procedure with the collars - At $3,7,11,15,22,26$ and 36 weeks post-attachment of the collars, dogs were sedated and put into nets with about 150 female $\mathrm{Lu}$. migonei. On weeks 4 , $8,12,16,22,27$ and 35 weeks post-attachment of the collars, tests were performed with $L u$. longipalpis using the same protocol. Each week, two sets of experiments were done; each night one deltamethrin-collared dog and one untreated-collared dog were tested, each group always using the same net. The pots were opened to release the flies into the net 10-15 min before the sedated dog was introduced. All experiments began at approximately 6 p.m. While the dog was added or removed, or while recapturing the flies, a fluorescent light was turned on to attract the flies to the top of the net. The dogs were exposed for a $2 \mathrm{~h}$ period and examined for sand flies on their fur before being removed. Dead flies knocked down in the net were collected and saved until the next day to see if they recovered, after which they were scored as engorged or non-engorged. Living flies were counted by inspection in the aspirator as they were replaced in the large pot. Accuracy of such inspection compared to inspection under the dissecting microscope was $99.5 \%$. Essentially, throughout the study no sand flies had taken a small blood meal that was detectable only by microscopy. Live flies were scored as engorged and not-engorged. These flies were held in the stock cage for $18 \mathrm{~h}$ after which dead flies were counted and scored as engorged and not-engorged and subtracted from the previous night's live totals. We recovered almost every fly from the nets. Percent engorgement was calculated as the ratio of the sum of all dead engorged flies after 2 and $20 \mathrm{~h}$ plus live engorged flies over the total of recovered sand flies. Percent mortality was calculated as the ratio of the sum of all dead sand flies after 2 and $20 \mathrm{~h}$ over the total of recovered sand flies.

Statistical analysis - Data used to produce Figs 1 and 2 were fitted on a repeated measure model, Stata (StataCorp 1999). In other statistical analysis Student's t test for unpaired samples was used.

\section{RESULTS}

The deltamethrin collars had a dramatic antifeeding effect on both species of sand flies. For Lu. longipalpis (Fig. 1A) inhibition of feeding was 99.3\% when tested 4 weeks after the application of the collars, $100 \%$ after 8 and 12 weeks and over $96 \%$ at 16 and 20 weeks. The anti-feeding effect 
went below $90 \%$ in one of two dogs tested at 27 weeks, but was still $94 \%$ at 35 weeks. Fitting the data on a repeated measure model (StataCorp 1999), the difference between the anti-feeding on deltamethrin collars and the untreated collars was highly significant $(\mathrm{p}<0.0005)$. The anti-feeding effect of the deltamethrin collars was still present after more than eight months (Table I).

The deltamethrin collars also caused mortality of $\mathrm{Lu}$. longipalpis (Fig. 1B). This ranged from $96 \%$ at 4 weeks to $35 \%$ at 35 weeks, gradually falling after 22 weeks. When the data were fitted in the model as above, the difference in the mortality of Lu. longipalpis with dogs with the deltamethrin collars compared to dogs with the untreated collars was highly significant ( $\mathrm{p}<0.0005)$. The mortality of Lu. longipalpis was recorded at $2 \mathrm{~h}$ and after $20 \mathrm{~h}$. In early experiments almost $100 \%$ of the sand flies with the dogs wearing deltamethrin collars were killed in the first $2 \mathrm{~h}$ (Table II). Throughout the study an average of $50 \%$ of the sand flies died within $2 \mathrm{~h}$. In contrast, over the whole study, only $1.1 \%$ of the sand flies with the untreated-collared dogs died at $2 \mathrm{~h}$. With time, the sand fly killing effect diminished considerably faster than the anti-feeding effect (Fig. 1). The majority of the sand flies that engorged on the deltamethrin collared dogs also died. This was $100 \%$ during the first 16 weeks, falling to $35 \%$ at 35 weeks. In contrast, the mortality of sand flies engorged on dogs with untreated collars ranged from $3.8 \%$ to $0.7 \%$ with an average of only $1.6 \%$. This difference was significant $(\mathrm{p}<0.01)$.

The deltamethrin collars also had a dramatic anti-feeding effect on Lu. migonei (Fig. 2A). Inhibition of feeding was $98.3 \%$ when tested 3 weeks after the application of the collars, $100 \%$ after 7 and 11 weeks, $99.3 \%$ at 15 weeks, $94.3 \%$ at 26 weeks and $91.8 \%$ at 36 weeks. Except for one test on $\operatorname{dog} \# 1$ at 26 weeks which showed $86.7 \%$ inhibition of feeding, the deltamethrin collars inhibited feeding by more than $90 \%$ throughout the 36 weeks of the study. We have no explanation for this single lower value except for biological variation. Fitting the data on a repeated measure model (StataCorp 1999) the difference between the deltamethrin collars and the untreated collars was highly significant $(\mathrm{p}<0.0005)$.

The deltamethrin collars also caused mortality of Lu. migonei (Fig. 2B). This ranged from $91 \%$ at 3 weeks to $46 \%$ at 36 weeks. Except for one test at 11 weeks, mortality gradually fell after 22 to 26 weeks. When the data were fitted in the model as above, the mortality of Lu. migonei with dogs with the deltamethrin collars compared to the untreated collars was highly significant $(\mathrm{p}<0.0005)$. As with $L u$. longipalpis, the sand fly killing effect dimin-
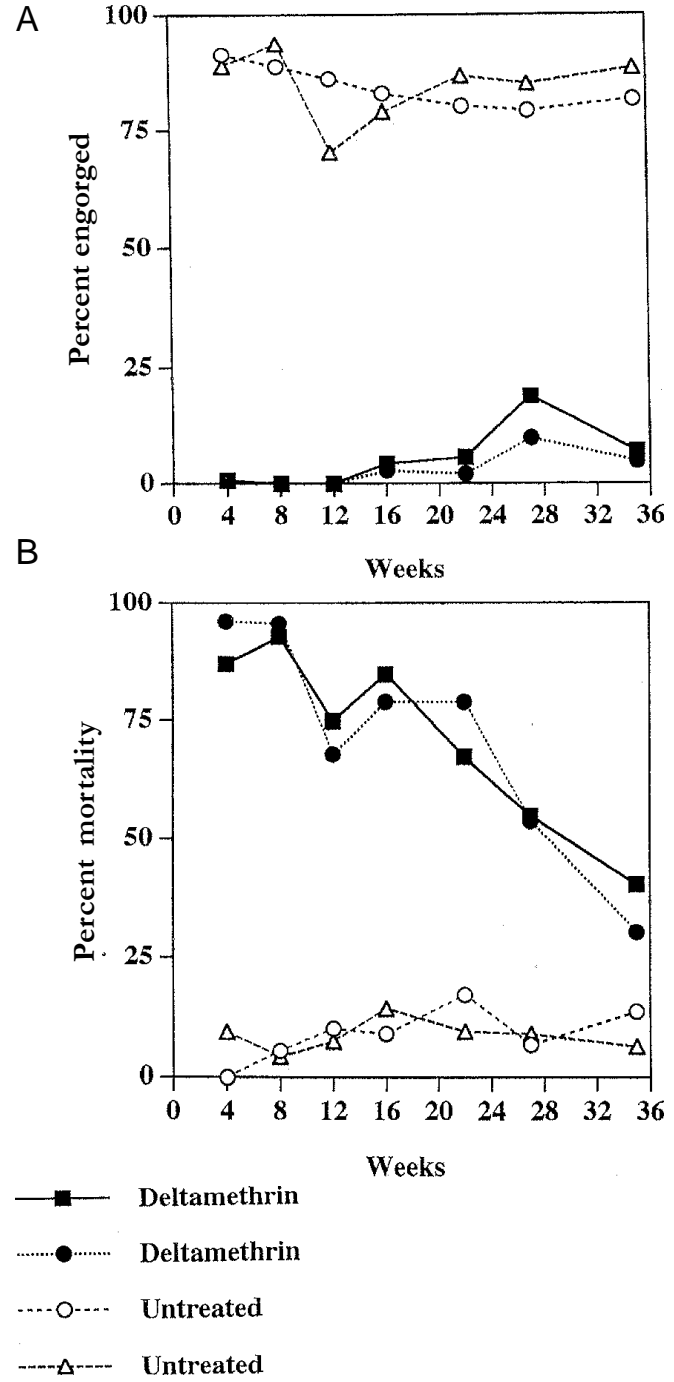

Fig. 1: deltamethrin collars prevent biting by Lutzomyia longipalpis and cause mortality. A: the anti-feeding effect is seen by the diminished engorgement in the dogs with the deltamethrin collars. Two of the four deltamethrin-collared dogs and two of the three untreated-collared dogs were tested each week thus the two lines. The identification of the dogs for any given week can be found on Table I; B: the mortality as assessed at $20 \mathrm{~h}$ includes sand flies that died after $2 \mathrm{~h}$.

ished considerably faster than the anti-feeding effect. The mortality ranged from $100 \%$ to $50 \%$ and was still $86 \%$ at 22 weeks. In contrast, the mortality of sand flies engorged on dogs with untreated collars ranged from 0 to $17 \%$. This difference was significant ( $\mathrm{p}<0.025)$. As with $L u$. longipalpis, the majority (55\%) of Lu. migonei placed with the deltamethrin collared dogs over the 36 weeks were dead when recovered at $2 \mathrm{~h}$. In contrast, over this period only $0.8 \%$ of $\mathrm{Lu}$. migonei with untreated collared dogs were dead at $2 \mathrm{~h}$. 
TABLE I

Results of experiments with Lutzomyia longipalpis exposed to dogs wearing a deltamethrin-impregnated collar

\begin{tabular}{|c|c|c|c|c|c|c|c|c|c|c|}
\hline \multirow[b]{2}{*}{ Week } & \multirow[b]{2}{*}{ Dog } & \multicolumn{2}{|c|}{ Dead flies after $2 \mathrm{~h}$} & \multicolumn{2}{|c|}{ Dead flies after $20 \mathrm{~h}$} & \multicolumn{2}{|c|}{ Live flies after $20 \mathrm{~h}$} & \multirow[b]{2}{*}{ Total $^{a}$} & \multirow[b]{2}{*}{$\begin{array}{c}\text { Percent } \\
\text { engorged }\end{array}$} & \multirow[b]{2}{*}{$\begin{array}{l}\text { Percent } \\
\text { mortality }\end{array}$} \\
\hline & & Engorged & $\begin{array}{c}\text { Not } \\
\text { engorged }\end{array}$ & Engorged & $\begin{array}{c}\text { Not } \\
\text { engorged }\end{array}$ & Engorged & $\begin{array}{c}\text { Not } \\
\text { engorged }\end{array}$ & & & \\
\hline 4 & 9 & 1 & 124 & 0 & 1 & 0 & 19 & 145 & 0.6 & 86.9 \\
\hline 4 & 10 & 1 & 142 & 0 & 2 & 0 & 6 & 151 & 0.6 & 96 \\
\hline 8 & 9 & 0 & 124 & 0 & 13 & 0 & 11 & 148 & 0 & 92.6 \\
\hline 8 & 10 & 0 & 141 & 0 & 6 & 0 & 7 & 154 & 0 & 95.5 \\
\hline 12 & 9 & 0 & 72 & 0 & 26 & 0 & 33 & 131 & 0 & 74.8 \\
\hline 12 & 10 & 0 & 78 & 0 & 13 & 0 & 43 & 134 & 0 & 67.9 \\
\hline 16 & 9 & 4 & 62 & 2 & 53 & 0 & 22 & 143 & 4.2 & 84.6 \\
\hline 16 & 10 & 3 & 103 & 1 & 8 & 0 & 31 & 146 & 2.7 & 78.8 \\
\hline 22 & 9 & 4 & 49 & 3 & 41 & 1 & 46 & 144 & 5.6 & 67.4 \\
\hline 22 & 10 & 1 & 78 & 0 & 36 & 2 & 29 & 146 & 2.1 & 78.8 \\
\hline 27 & 9 & 3 & 18 & 7 & 51 & 17 & 48 & 144 & 18.8 & 54.9 \\
\hline 27 & 10 & 1 & 34 & 4 & 39 & 9 & 58 & 145 & 9.6 & 53.8 \\
\hline 35 & 9 & 0 & 22 & 2 & 35 & 8 & 79 & 146 & 6.9 & 40.4 \\
\hline 35 & 10 & 3 & 28 & 1 & 12 & 3 & 98 & 145 & 4.8 & 30.3 \\
\hline Total & - & 21 & 1,075 & 20 & 336 & 40 & 530 & 2,022 & & \\
\hline
\end{tabular}

$a:$ total $=2 \mathrm{~h}+20 \mathrm{~h}$

TABLE II

Results of experiments with Lutzomyia longipalpis exposed to dogs wearing a control collar

\begin{tabular}{|c|c|c|c|c|c|c|c|c|c|c|}
\hline \multirow[b]{2}{*}{ Week } & \multirow[b]{2}{*}{ Dog } & \multicolumn{2}{|c|}{ Dead flies after $2 \mathrm{~h}$} & \multicolumn{2}{|c|}{ Dead flies after $20 \mathrm{~h}$} & \multicolumn{2}{|c|}{ Live flies after $20 \mathrm{~h}$} & \multirow[b]{2}{*}{ Total $^{a}$} & \multirow[b]{2}{*}{$\begin{array}{c}\text { Percent } \\
\text { engorged }\end{array}$} & \multirow[b]{2}{*}{$\begin{array}{l}\text { Percent } \\
\text { mortality }\end{array}$} \\
\hline & & Engorged & $\begin{array}{c}\text { Not } \\
\text { engorged }\end{array}$ & Engorged & $\begin{array}{c}\text { Not } \\
\text { engorged }\end{array}$ & Engorged & $\begin{array}{c}\text { Not } \\
\text { engorged }\end{array}$ & & & \\
\hline 4 & 7 & 0 & 0 & 0 & 0 & 135 & 13 & 148 & 91.2 & 0 \\
\hline 4 & 5 & 0 & 1 & 2 & 11 & 131 & 5 & 150 & 88.7 & 9.3 \\
\hline 8 & 5 & 0 & 0 & 2 & 6 & 129 & 11 & 148 & 88.5 & 5.4 \\
\hline 8 & 6 & 0 & 0 & 1 & 6 & 156 & 5 & 168 & 93.5 & 4.2 \\
\hline 12 & 6 & 0 & 2 & 1 & 12 & 127 & 7 & 149 & 85.9 & 10.1 \\
\hline 12 & 6 & 0 & 0 & 3 & 9 & 110 & 39 & 161 & 70.2 & 7.5 \\
\hline 16 & 7 & 0 & 2 & 4 & 7 & 116 & 16 & 145 & 82.8 & 9 \\
\hline 16 & 5 & 0 & 3 & 5 & 13 & 111 & 15 & 147 & 78.9 & 14.3 \\
\hline 22 & 5 & 1 & 0 & 2 & 22 & 114 & 7 & 146 & 80.1 & 17.1 \\
\hline 22 & 6 & 0 & 2 & 0 & 12 & 128 & 6 & 148 & 86.5 & 9.5 \\
\hline 27 & 7 & 1 & 0 & 2 & 7 & 115 & 24 & 149 & 79.2 & 6.7 \\
\hline 27 & 5 & 0 & 2 & 2 & 9 & 121 & 11 & 145 & 84.8 & 9 \\
\hline 35 & 5 & 0 & 3 & 0 & 17 & 119 & 7 & 146 & 81.5 & 13.7 \\
\hline 35 & 6 & 0 & 2 & 2 & 5 & 125 & 10 & 144 & 88.2 & 6.3 \\
\hline Total & - & 2 & 17 & 26 & 136 & 1,737 & 176 & 2,094 & & \\
\hline
\end{tabular}

$a:$ total $=2 \mathrm{~h}+20 \mathrm{~h}$

Examining all the data to 35 weeks, of the total of 2,022 Lu. longipalpis recovered from the nets with the deltamethrin collared dogs, 81 or $4 \%$ were engorged (Table I). Further, only $2 \%$ of the $L u$. longipalpis that became engorged in the nets with the deltamethrin-collared dogs during the 35 weeks remained alive. In contrast, of the 2,094 $\mathrm{Lu}$. longipalpis recovered from the nets with the untreated collared dogs, 1,737 or $83 \%$ were engorged and $98.4 \%$ of these engorged flies remained alive.
During this period of study, $54 \%$ of the sand flies with the deltamethrin-collared dogs died during the first $2 \mathrm{~h}$ and only $2 \%$ of these dead flies were engorged. In contrast, only $10 \%$ of the sand flies in the nets with the untreated- collared dogs died at $2 \mathrm{~h}$. Of the 2,094 sand flies in these nets, only 19 sand flies $(1.1 \%)$ died after $2 \mathrm{~h}$ and only 2 of the these flies were engorged.

Similarly, all the data to 36 weeks showed that 21 out of the 2,034 (1\%) Lu. migonei recovered 
A

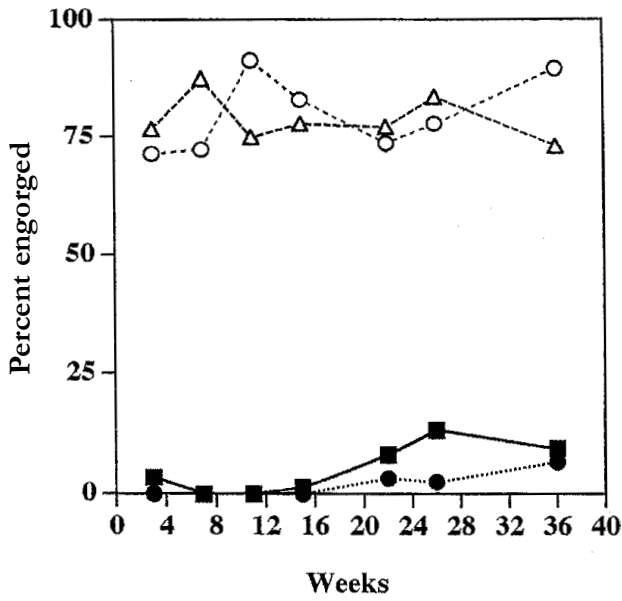

B

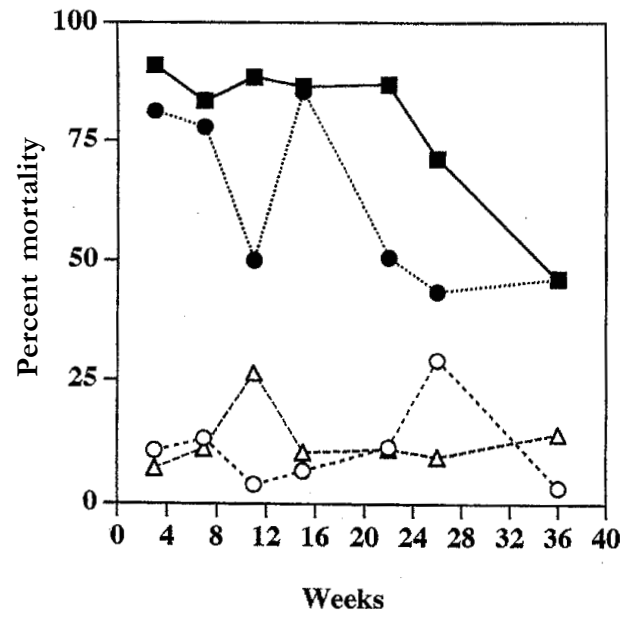

$\longrightarrow$ Deltamethrin

............... Deltamethrin

-...-.... Untreated

-.-- Untreated

Fig. 2: deltamethrin collars prevent biting by Lutzomyia migonei and cause mortality. A: the anti-feeding effect is seen by the diminished engorgement in the dogs with the deltamethrin collars. Two of the four deltamethrin-collared dogs and two of the three untreated-collared dogs were tested each week. The identification of the dogs for any given week can be found on Table II; B: the mortality as assessed at $20 \mathrm{~h}$ includes sand flies that died after $2 \mathrm{~h}$.

from the tents with the deltamethrin- collared dogs were engorged and living compared to 1555 out of $2,035(76 \%)$ from the tents with the untreated collared dogs (Table III). Furthermore, 69\% of the 68 sand flies that engorged on the deltamethrincollared dogs died during this period (Table IV).

\section{DISCUSSION}

Killick-Kendrick et al. (1997) showed that deltamethrin-impregnated collars prevented the feeding of laboratory reared $P$. perniciosus on beagles by an average of $94 \%$ over 34 weeks. Only $0-12 \%$ of the recaptured sand flies had fed on deltamethrin collared dogs compared to 55-95\% that had fed on the collarless dogs. Furthermore, immediately after the dogs were removed from the nets, $21-60 \%$ of the flies from the collared dogs were dead compared to $0-12 \%$ from the controls. Lucientes (1999) has confirmed these findings also using laboratory-reared $P$. perniciosus and beagles. He found that $85-98 \%$ of the deltamethrin-collared dogs were protected from bites of sand flies compared to dogs with untreated collars lacking deltamethrin. The lethal effect of the deltamethrin collars was about $50 \%$. His study was carried out over 26 weeks. Recently, Halbig et al. (2000) reported on a study in Iran using the same deltamethrin collars and wild-caught $P$. papatasi confined with large guard dogs. They tested the anti-feeding and killing effect before attachment and after the collars had been on the dogs for eight days. It rose from 11 to $51 \%$. Sand fly mortality, however, did not differ, being $17 \%$ before and $18 \%$ eight days after attaching the collars.

During the development of the impregnated PVC collars, it was found that the deltamethrin was released slowly over several months from the collars (Killick-Kendrick et al. 1997). As deltamethrin is lipophilic like all synthetic pyrethroids (Miller \& Salago 1985), it spreads in the dermal secretions over most of the body of the dog. This explains the delay in reaching its maximum efficacy demonstrated by the tests in KillickKendrick's study at one week, and probably explains the lower efficacy and lack of lethal effect of the deltamethrin collars in the study on $P$. papatasi done on day 8 . Studies by the producers of the collars have also shown that their activity resists both rain and sun. Indeed, they were effective on dogs that were exposed to rain (KillickKendrick et al. 1997).

As different species of sand flies, as well as mosquitoes, show different behavior and insecticide susceptibility (Killick-Kendrick 1999), it was important to determine whether if neotropical Lutzomyia spp. were as susceptible as Phlebotomus spp. to the deltamethrin from the collars. Indeed, whereas pyrethroid impregnated clothing was found to protect against Phlebotomus spp. in the Sinai desert (Dees et al. 1985), it protected poorly against Lutzomyia spp. in Panama (Schreck et al. 1982). Our studies show that Lu. longipalpis and Lu. migonei are at least as susceptible as the two previously tested phlebotomines to the anti-feed- 
TABLE III

Results of experiments with Lutzomyia migonei exposed to dogs wearing a deltamethrin-impregnated collar

\begin{tabular}{|c|c|c|c|c|c|c|c|c|c|c|}
\hline \multirow[b]{2}{*}{ Week } & \multirow[b]{2}{*}{ Dog } & \multicolumn{2}{|c|}{ Dead flies after $2 \mathrm{~h}$} & \multicolumn{2}{|c|}{ Dead flies after $20 \mathrm{~h}$} & \multicolumn{2}{|c|}{ Live flies after $20 \mathrm{~h}$} & \multirow[b]{2}{*}{ Total $^{a}$} & \multirow[b]{2}{*}{$\begin{array}{c}\text { Percent } \\
\text { engorged }\end{array}$} & \multirow[b]{2}{*}{$\begin{array}{c}\text { Percent } \\
\text { mortality }\end{array}$} \\
\hline & & Engorged & $\begin{array}{c}\text { Not } \\
\text { engorged }\end{array}$ & Engorged & $\begin{array}{c}\text { Not } \\
\text { engorged }\end{array}$ & Engorged & $\begin{array}{c}\text { Not } \\
\text { engorged }\end{array}$ & & & \\
\hline 3 & 1 & 0 & 50 & 3 & 79 & 2 & 11 & 145 & 3.5 & 91 \\
\hline 3 & 8 & 0 & 114 & 0 & 4 & 0 & 27 & 145 & 0 & 81.4 \\
\hline 7 & 1 & 0 & 106 & 0 & 6 & 0 & 22 & 134 & 0 & 83.6 \\
\hline 7 & 8 & 0 & 96 & 0 & 17 & 0 & 32 & 145 & 0 & 77.9 \\
\hline 11 & 1 & 0 & 102 & 0 & 22 & 0 & 16 & 140 & 0 & 88.6 \\
\hline 11 & 8 & 0 & 49 & 0 & 23 & 0 & 72 & 144 & 0 & 50 \\
\hline 15 & 1 & 2 & 118 & 0 & 9 & 0 & 20 & 149 & 1.3 & 86.6 \\
\hline 15 & 8 & 0 & 96 & 0 & 33 & 0 & 22 & 151 & 0 & 85.4 \\
\hline 22 & 1 & 8 & 86 & 2 & 31 & 2 & 17 & 146 & 8.2 & 87 \\
\hline 22 & 8 & 2 & 50 & 1 & 23 & 2 & 72 & 150 & 3.3 & 50.7 \\
\hline 26 & 1 & 11 & 79 & 6 & 0 & 1 & 38 & 135 & 13.3 & 71.1 \\
\hline 26 & 8 & 2 & 44 & 0 & 22 & 2 & 86 & 156 & 2.6 & 43.6 \\
\hline 36 & 1 & 2 & 43 & 3 & 20 & 9 & 70 & 147 & 9.5 & 46.3 \\
\hline 36 & 8 & 7 & 54 & 0 & 7 & 3 & 76 & 147 & 6.8 & 46.3 \\
\hline Total & - & 34 & 1,087 & 15 & 296 & 21 & 581 & 2,034 & & \\
\hline
\end{tabular}

$a:$ total $=2 \mathrm{~h}+20 \mathrm{~h}$

TABLE IV

Results of experiments with Lutzomyia migonei exposed to dogs wearing a control collar

\begin{tabular}{|c|c|c|c|c|c|c|c|c|c|c|}
\hline \multirow[b]{2}{*}{ Week } & \multirow[b]{2}{*}{ Dog } & \multicolumn{2}{|c|}{ Dead flies after $2 \mathrm{~h}$} & \multicolumn{2}{|c|}{ Dead flies after $20 \mathrm{~h}$} & \multicolumn{2}{|c|}{ Live flies after $20 \mathrm{~h}$} & \multirow[b]{2}{*}{ Total $^{a}$} & \multirow[b]{2}{*}{$\begin{array}{l}\text { Percent } \\
\text { engorged }\end{array}$} & \multirow[b]{2}{*}{$\begin{array}{l}\text { Percent } \\
\text { mortality }\end{array}$} \\
\hline & & Engorged & $\begin{array}{c}\text { Not } \\
\text { engorged }\end{array}$ & Engorged & $\begin{array}{c}\text { Not } \\
\text { engorged }\end{array}$ & Engorged & $\begin{array}{c}\text { Not } \\
\text { engorged }\end{array}$ & & & \\
\hline 3 & 5 & 0 & 1 & 3 & 11 & 96 & 28 & 139 & 71.2 & 10.8 \\
\hline 3 & 6 & 0 & 1 & 0 & 9 & 105 & 22 & 137 & 76.6 & 7.3 \\
\hline 7 & 6 & 0 & 0 & 2 & 17 & 102 & 23 & 144 & 72.2 & 13.2 \\
\hline 7 & 7 & 0 & 2 & 2 & 12 & 123 & 4 & 143 & 87.4 & 11.2 \\
\hline 11 & 7 & 0 & 1 & 0 & 5 & 136 & 7 & 149 & 91.3 & 4 \\
\hline 11 & 5 & 0 & 2 & 8 & 32 & 111 & 6 & 159 & 74.8 & 26.4 \\
\hline 15 & 5 & 0 & 0 & 2 & 8 & 120 & 17 & 147 & 83 & 6.8 \\
\hline 15 & 6 & 0 & 0 & 6 & 9 & 106 & 23 & 144 & 77.8 & 10.4 \\
\hline 22 & 6 & 1 & 5 & 3 & 8 & 104 & 26 & 147 & 73.5 & 11.6 \\
\hline 22 & 7 & 0 & 0 & 0 & 16 & 111 & 17 & 144 & 77.1 & 11.1 \\
\hline 26 & 5 & 0 & 0 & 26 & 16 & 86 & 16 & 144 & 77.8 & 29.8 \\
\hline 26 & 6 & 0 & 1 & 2 & 11 & 120 & 12 & 146 & 83.6 & 9.6 \\
\hline 36 & 7 & 0 & 0 & 1 & 4 & 128 & 11 & 144 & 89.6 & 3.5 \\
\hline 36 & 5 & 0 & 2 & 1 & 18 & 107 & 20 & 148 & 73 & 14.2 \\
\hline Total & & 1 & 15 & 56 & 176 & 1,555 & 232 & 2,035 & & \\
\hline
\end{tabular}

$a:$ total $=2 \mathrm{~h}+20 \mathrm{~h}$

ing effects of the deltamethrin collars and much more susceptible to the lethal effect. Furthermore, as we used mongrel dogs, our studies show that the anti-feeding and killing effect of the deltamethrin collars are not limited to beagles.

The anti-feeding effect appears to need less pyrethroid than the killing effect as is seen by the more rapid decrease in the lethal effect compared to anti-feeding effect with time in our study, and to the absence of a lethal effect despite an anti- feeding effect in the study after only eight days with $P$. papatasi. This is consistent with the findings of Wu et al. (1991) who showed strong antifeeding effects of deltamethrin on impregnated gauze on Culex quinquefasciatus well below doses recommended to kill the mosquito.

The anti-feeding effects we observed (determined by the lack of engorgement), is probably due to the neurotoxic effects of pyrethroids (Soderlund \& Bloomquist 1989). Alterations of the 
voltage-sensitive sodium channel is the principle molecular target site for all pyrethroids in insects and mammals, although it is probably not the only site involved in intoxication. Insect neurosecretory neurons are sensitive to low concentrations of pyrethroids and disruption of the neuroendocrine systems has also been implicated as contributing to pyrethroid intoxication.

The finding in our study that only $1 \%$ of the few sand flies that have fed live after the first bite should significantly reduce the circulation of the Leishmania parasite when the dog is the major reservoir host. This is especially true since flies have to bite twice to transmit the Leishmania parasite to the reservoir host and the collars should greatly reduce the passage of the parasite from dog to dog as well as from dog to man. Indeed, it is probable the risk of infection to man where the dog is the predominant reservoir host would be reduced significantly or fall to zero (Killick-Kendrick 1999).

These deltamethrin collars should not have the untoward effect of causing sand flies to bite humans instead of dogs. Once having gone to the dogs, the majority of flies would die before getting a blood meal and thus very few of the remaining living sand flies would be infected.

A field trial is about to start to find out whether the collars are effective on dogs in their natural habitat. If they are, the deltamethrin collars would appear to be an excellent and cost effective strategy for the control of leishmaniasis where the dog is the main animal reservoir. Use of collars should be much more acceptable to the population than the current strategy of killing seropositive dogs. The deltamethrin collars, however, must first be shown to be effective in the field, i.e. they should dramatically decrease the seroconversion of seronegative dogs in the endemic areas. The effect of collaring dogs on the incidence of human cases of VL must be also assessed. The collars could also be used to determine how important the dog is in the transmission of the parasites in various other clinical forms of leishmaniasis where dogs have been postulated to be the natural reservoir host.

\section{ACKNOWLEDGEMENTS}

To Prof. Marcelo Pagano from the Department of Biostatistics, Harvard School of Public Health for carrying out the statistical analysis using a repeated measure model, to Gina Monteforte for making the figures, to Jose Valderi Souza, Afonso Celso Gadelha, Ana Claudia Regazzi for their help with the sand fly colonies, and to Maria Nogueira Evangelista for watching over the kennels by her house.

\section{REFERENCES}

Adler S, Theodor O 1932. Investigations on Mediterranean kala-azar. VI - Canine visceral leishmaniasis. Proc R Soc Lond 110: 402-412.
Akhavan D 1996. Análise de custo-efetividade do componente de leishmaniose no projeto de controle de doenças endêmicas no nordeste do Brasil. Rev Patol Trop 25: 203-252.

Alexander B 2000. Sampling methods for phlebotomine sand flies. Med Vet Entomol 14: 109-122

Ashford DA, David JR, Freire M, David R, Sherlock I, Eulalio MC, Sampaio DP, Badaro R 1998. Studies on control of visceral leishmaniasis: impact of dog control on canine and human visceral leishmaniasis in Jacobina, Bahia, Brazil. Am J Trop Med Hyg 59: 53-57.

Bettini S, Gradoni L 1986. Canine leishmaniasis in the Mediterranian area and its implications for human leishmaniasis. Insect Sci Appli 7: 241-245.

Bettini S, Contini C, Atzeni MC, Tocco G 1986. Leishmaniasis in Sardinia. I. Observations on a larval breeding site of Phlebotomus perniciosus, Phlebotomus perfiliewi perfiliewi and Sergentomyia minuta (Diptera: Psychodidae) in the canine leishmaniasis focus of Soleminis (Cagliari). Ann Trop Med Parasitol 80: 307-315.

Dees WH, Overington KR, Karuru ER, Helmy L, Schreck CE 1985. Preliminary investigations of protective clothing and repellents used against medically important arthropods in the Sinai Desert. Meeting Proceedings, New Jersey Mosquito Control Association, Atlantic City, p. 239-241.

Grimaldi G, Tesh RB, McMahon-Pratt D 1989. A review of the geographic distribution and epidemiology of leishmaniasis in the New World. Am J Trop Med Hyg 41: 687-725.

Halbig P, Hodjati MH, Mazloumi-Gavagni AS, Mohite H, Davies CR 2000. Further evidence that deltamethrin-impregnated collars protect domestic dogs from sandfly bites. Med Vet Entomol 14: 223-226.

Jarvis EK, Rutledge LC 1992. Laboratory observations on mating and lek-like aggregations in Lutzomyia longipalpis (Diptera: Psychodidae). J Med Entomol 29: 171-177.

Killick-Kendrick R 1999. Anti-feeding effects of synthetic pyrethroids against phlebotomine sand flies and mosquitoes, and the prospects of controlling canine leishmaniasis with deltamethrin-impregnated ProtectorBands (Scalibor®). In R Killick-Kendrick, $\mathrm{Ca}$ nine Leishmaniasis: an Update, Proceedings of a Canine Leishmaniasis Forum, Barcelona (Sitges), 28-31 January, 1999, Wiesbaden: Hoechst Roussel Vet, p. 82-88 (available gratis from jac. bergman@intervet.com).

Killick-Kendrick R, Killick-Kendrick M, Focheux MC, Dereure J, Puech MP, Cadiergues MC 1997. Protection of dogs from bites of phlebotomine sandflies by deltamethrin collars for control of canine leishmaniasis. Med Vet Entomol 11: 105-111.

Killick-Kendrick R, Leaney AJ, Ready PD 1977. The establishment, maintenance and productivity of a laboratory colony of Lutzomyia longipalpis (Diptera: Psychodidae). J. Med Entomol 13: 429-440.

Lucientes J 1999. Laboratory observations on the protection of dogs from bites of Phlebotomus perniciosus with Scalibor ${ }^{\circledR}$ ProtectorBands ${ }^{\circledR}$ : preliminary results. In R Killick-Kendrick, Canine 
Leishmaniasis: an Update, Proceedings of a Canine Leishmaniasis Forum, Barcelona (Sitges), 28-31 January, 1999, Wiesbaden: Hoechst Roussel Vet, p. 92-94 (available gratis from jac. bergman@intervet.com).

Marzochi MCA, Marzochi KBF 1994. Tegumentary and visceral leishmaniasis in Brazil - Emerging anthropozoonosis and possibilities of their control. Cad Saúde Pública 10 (Suppl. 2): 359-375.

Mauricio IL, Howard MK, Stothard JR, Miles MA 2000a. Genomic diversity in the Leishmania donovani complex. Parasitology 119: 237-246.

Mauricio IL, Stothard R, Miles MA 2000b, The strange case of Leishmania chagasi. Parasitol Today 16: 188-189.

Miller TA, Salago VL 1985. The mode of action of pyrethroids on insects. In JP Leahey, The Pyrethroid Insecticides, Taylor and Francis, London, p. 43-97.

Murray CJL, Lopez AD 1996. The Global Burden of Disease, HSPH/WHO/Harvard University Press, Boston, p. 264, 348.

Nicolle G, Comte M 1908. Recherches sur la kala-azar enterprises a l'Institut Pasteur de Tunis. IV. Origine canine du kala-azar. Arch Inst Pasteur Tunis 3: 5962.

Schreck CE, Kline DL, Chaniotis BN, Wilkinson N, McGovern TP, Weidhaas DE 1982. Evaluation of personal protection methods against phlebotomine sand flies including vectors of leishmaniasis in Panama. Am J Trop Med Hyg 31: 1046-1053.

Shaw JJ, Lainson R 1987. Ecology and epidemiology: New World. In R Killick-Kendrick, W Peters (eds), The Leishmaniases in Biology and Medicine, Academic Press, London, p. 365.

Sherlock IA, Pessoa SB 1964. Métodos practicos para a captura de flebótomos. Rev Bras Biol 24: 331-340.

Soderlund DM, Bloomquist JR 1989. Neurotoxic actions of pyrethroid insecticides. Annu Rev Entomol 34: 77-96.

StataCorp 1999. Stata Statistical Software: Release 6.0, College Station, TX: Stata Corporation. The program used within this package was $\mathrm{glm}$.

Wu Neg, Xiao Yan, Chen D, Huan F 1991. Laboratory evaluation of efficacy of bednets impregnated with pyrethroids. J Mosquito Control Assoc 7: 294-298. 